\title{
Study of glycolic acid and salicylic acid peels as a sole therapy in treatment of acne vulgaris
}

\author{
Sharma $\mathbf{P}^{1}$, Shah $\mathrm{A}^{2}$, Dhillon $\mathrm{AS}^{3}$ \\ ${ }^{1}$ Dr. Parikshit Sharma, Associate Professor, ${ }^{2}$ Dr. Akhil Shah, Assistant Professor, ${ }^{3}$ Dr. Alamjit singh Dhillon, P.G. \\ Resident; all authors are affiliated with Department of Skin \& VD, Index Medical College, Indore, MP, India
}

Address for correspondence: Dr. Parikshit Sharma, Email: drparikshitsharma@ gmail.com

\begin{abstract}
Introduction: Acne vulgaris is a very common physiological condition of adolescents, better regarded as a disease due to some inflammatory component. In spite of various modalities of treatment available, it still poses a therapeutic problem to the dermatologists. The advent of new and potent topical therapeutic modalities such as chemical peeling has resulted in significant improvement in the treatment of acne. This study aims to observe the therapeutic response of two chemical peels, $35 \%$ glycolic acid and 30\% salicylic acid on active acne lesions, when given as monotherapy. Materials \& Methods: A total of 200 patients of either sex with acne were randomly selected and enrolled for the study, two groups of 100 patients each were made of 35\% GA and 30\% SA respectively. No other concurrent therapy was given. Individual lesions of each type were calculated before and after completing 6 treatment sessions and the numbers were observed to know the effect of both the acids on different types of lesions. Results: After 6 sessions, in the glycolic acid group the reduction in average number of comedones was $88.45 \%$, inflammatory papules $88.65 \%$, pustules $89.62 \% \&$ nodules/cysts $83.33 \%$. Whereas, in the Salicylic acid group, the reduction was $89.00 \%, 90.36 \%$, was $84.87 \% \& 100 \%$ in comedones, papules, pustules \& nodules/cysts respectively. Conclusion: In our observation, both $35 \%$ glycolic acid and $30 \%$ salicylic acid are significantly effective as monotherapy in patients of acne. They not only clear the acne lesions but also show improvement in post acne scar and hyperpigmentation to some extent.
\end{abstract}

Keywords: Acne vulgaris, Chemical peeling, Glycolic acid, Salicylic acid

\section{Introduction}

Dermatologists have used various peeling agents for decades. This concept dates back to Roman times. Superficial and medium depth skin peeling with trichloroacetic acid has been a well documented therapy in United States since at least till 1960, when Dr. Baker and Gordon pioneered chemical peeling with phenol in the year 1961[1]. Chemical peeling is a technique used to improve the appearance of the skin. A chemical solution is applied to the skin, which causes it to separate, peel off and allows new skin to regenerate. The new skin is smoother and less wrinkled than the old skin, and may also be more even in color. Chemical peeling is one of the latest treatments available for many dermatological conditions in modern era. Superficial and medium depth skin peels can create

Manuscript received: $16^{\text {th }}$ November 2016

Reviewed: $24^{\text {th }}$ November 2016

Author Corrected: $01^{\text {st }}$ December 2016

Accepted for Publication: 09 $9^{\text {th }}$ December 2016 dramatic improvement in the skin but the results were not long lasting as those with phenol peels. The use of retinoic acid, alpha and beta hydroxy acids, broadspectrum sunscreens and skin bleaches as part of a postpeel maintenance programme has allowed patients maintain the improvement in skin for longer period of time [2]. Acne vulgaris is a very common physiological condition of adolescents but it is better regarded as a disease due to inflammatory component and the disfigurements it produces on socially and psychologically most important body region. Even though various modalities of treatment are available for acne, it still poses a therapeutic problem to the attending dermatologist.

The advent of new and potent topical therapeutic agents such as chemical peeling has resulted in significant improvement in the treatment of acne. This study 
attempts to find the therapeutic response of the two chemical peels, $35 \%$ glycolic acid and 30\%salicylic acid on active acne lesions when given as monotherapy.

\section{Materials \& Methods}

The study was conducted at skin OPD of Index Medical College over a period of 10 months from May 2014 to February 2015. A total of 200 patients of either sex with acne vulgaris were enrolled for the above study. The study was prospective observational study.

\section{Inclusion Criteria}

1) Patients of either sex with acne vulgaris.

2) Age between 15 to 30 years.

3) Patients with lesions limited to face only.

4) Both new and already treated patients who have stopped therapy for at least 4 weeks.

\section{Exclusion Criteria}

1) Taking any acne inducing drugs.

2) Hormonal acne (patient with known h/o PCOD, menstrual irregularities and patient on hormonal therapy for acne)

3) Patients with occupations prone to develop acne.

Treatment Protocol and Methodology: Patients selected were informed the nature of the study and written consent was obtained from the patients. The Demographic data such as age and sex of the selected patients, and duration of the disease were taken. Other history, like family history of acne, use of cosmetics, treatment history like topical and oral treatment for acne, topical steroids and other precipitating factors such as home remedies (mud, curd, honey etc.) were noted.

\section{Results}

Age distribution: Majority of patients (95\%) were in the age group of 15-25 years which is in accordance with the well known fact that acne is the disease of the young and adolescents. (Table 1)

Table-1: Age Distribution

\begin{tabular}{|l|l|l|}
\hline \multirow{2}{*}{$\begin{array}{l}\text { Age Groups } \\
\text { (years) }\end{array}$} & No. of Cases \\
\cline { 2 - 3 } & No. & Percentage \\
\hline $15-20$ & 86 & $43 \%$ \\
\hline $21-25$ & 104 & $52 \%$ \\
\hline $26-30$ & 10 & $5 \%$ \\
\hline Total & 200 & $100 \%$ \\
\hline
\end{tabular}

Patients were subjected for general and systemic examination. A thorough Dermatological examination was done and other existing Dermatological lesions apart from acne were recorded. Dermatological examination for acne included the grading of acne as 1 to 4 as follows [3]

Grade 1 - only comedones few papules

Grade 2 - inflammatory papules and comedones

Grade 3 - predominant pustules, papules and comedones

Grade 4 - nodulocystic

A total of 200 patients of either sex with acne were randomly selected and enrolled for the study, two groups of 100 patients each were made of $35 \% \mathrm{GA}$ and $30 \%$ SA respectively. No other concurrent therapy was given. Individual lesions of each type were calculated before and after completing 6 treatment sessions and the numbers were observed to know the effect of both the acids on different types of lesions.

Clinical photographs of the face were taken before commencement of therapy and after completion of 6 sessions therapy. 100 patients who fulfilled the inclusion criteria were selected and were applied $35 \%$ glycolic acid on the face and similarly 100 other patients were applied $30 \%$ salicylic acid on the face every 15 days for a total of 6 sessions. Patients were advised to avoid cosmetics and were started on priming with retinoic acid at night for 1 week.

The results were noted by counting the individual lesions (comedones, papules, pustules, nodules \& cysts) before and at the end of the treatment. The Mean standard deviation of individual lesions were calculated and paired ' $\mathrm{t}$ ' test was performed with $\mathrm{P}$ value $<0.05$ considered statistically significant. 
Sex distribution: $64 \%$ of our patients were females and $36 \%$ were males. (Table 2)

Table-2: Sex Distribution

\begin{tabular}{|l|l|l|}
\hline \multirow{2}{*}{ Sex } & No. of Cases & Percentage \\
\cline { 2 - 3 } & No. & $36 \%$ \\
\hline Male & 72 & $64 \%$ \\
\hline Female & 128 & $100 \%$ \\
\hline Total & 200 & 100 \\
\hline
\end{tabular}

Grading of acne vulgaris: Comedonal acne was the most common morphological variant of acne (64\%) followed by papules, pustules and nodules/cysts (Table 3). Table 4 demonstrate response with $35 \%$ glycolic acid peels.

Table 3: Acne grading

\begin{tabular}{|l|l|l|}
\hline Grade & No. & Percentage \\
\hline Grade I & 128 & $64 \%$ \\
\hline Grade II & 22 & $11 \%$ \\
\hline Grade III & 38 & $19 \%$ \\
\hline Grade IV & 12 & $6 \%$ \\
\hline
\end{tabular}

Table-4: Effect of peel on acne lesion observed in glycolic acid peels

\begin{tabular}{|l|l|l|l|}
\hline Morphology of Lesions & $\begin{array}{l}\text { Glycolic Acid Session I } \\
\text { (Mean) }\end{array}$ & $\begin{array}{l}\text { Glycolic Acid } \\
\text { Session VI (Mean) }\end{array}$ & $\begin{array}{l}\text { Mean Percentage of } \\
\text { Reduction }\end{array}$ \\
\hline Comedones & $5.37 \pm 2.4$ & $0.62 \pm 0.79$ & $88.45 \pm 0.15$ \\
\hline papules & $5.11 \pm 2.35$ & $0.58 \pm 0.7$ & $89.16 \pm 0.15$ \\
\hline Pustules & $1.06 \pm 1.78$ & $0.11 \pm 0.37$ & $31.47 \pm 0.45$ \\
\hline Nodules & $0.06 \pm 0.24$ & $0.01 \pm 0.1$ & $5.0 \pm 0.22$ \\
\hline
\end{tabular}

Effect of peel on acne lesions observed in 100 patients of glycolic acid group.

1) There was reduction of both non- inflammatory and inflammatory acne.

2) After 6 sessions the reduction in average number of
a) Comedones was $88.45 \%, \mathrm{P}$ value $=0.000$ (highly significant)
b) Inflammatory papules was $88.65 \%, \mathrm{P}$ value $=0.000$ (highly significant)
c) Pustules was $89.62 \%$ with $\mathrm{P}$ value $=0.000$ (highly significant) \&
d) Nodules/cysts were reduced by $83.33 \%$ with $\mathrm{P}$ value $=0.25$ ( not significant )

3) GA showed improvement in superficial scarring and skin texture. It also resolved post acne hyperpigmentation to some extent.

Table 5 demonstrate response with $30 \%$ salicylic acid peels.

Table-5: Effect of peel on acne lesion observed in salicylic acid peels

\begin{tabular}{|l|l|l|l|}
\hline Morphology of Lesions & $\begin{array}{l}\text { Salicylic Acid Session } \\
\text { I (Mean) }\end{array}$ & $\begin{array}{l}\text { Salicylic Acid Session VI } \\
\text { (Mean) }\end{array}$ & $\begin{array}{l}\text { Mean Percentage of } \\
\text { Reduction }\end{array}$ \\
\hline Comedones & $5.18 \pm 2.43$ & $0.57 \pm 0.71$ & $89.00 \pm 0.21$ \\
\hline Papules & $5.29 \pm 2.43$ & $0.51 \pm 0.73$ & $90.36 \pm 0.13$ \\
\hline Pustules & $1.19 \pm 2.05$ & $0.18 \pm 0.45$ & $28.3 \pm .42$ \\
\hline Nodules & $0.04 \pm 0.24$ & $0.00 \pm 0.00$ & $.03 . \pm 0.4$ \\
\hline
\end{tabular}

Effect of peel on acne lesions observed in 100 patients in salicylic acid group,

1) Reduction of both non-inflammatory and inflammatory acne was observed. 
2) After 6 sessions, the reduction in average number of

a) Comedones was $89.00 \%$ with $P$ value of 0.000 (highly significant)

b) Inflammatory papules were reduced by $90.36 \%$, with $\mathrm{P}$ value 0.000 (highly significant)

c) Pustules was $84.87 \%$ with P value of 0.000 (highly significant)

d) Nodules were reduced by $100 \%$. With P value of 0.41 (not significant)

3) SA showed improvement in post acne hyperpigmentation and overall appearance. It also resolved post acne scarring to some extent.

4) Side effects like Erythema-95\%, burning sensation-100\%, Dryness -98\% \& Peeling - 95\% were more with salicylic acid peel group in comparison to glycolic acid peel group.

Table-6: Response to treatment

\begin{tabular}{|c|c|c|c|c|c|}
\hline \multirow[t]{2}{*}{ Lesions } & \multirow[t]{2}{*}{$\begin{array}{l}\text { \% of reduction with } \\
35 \% \text { GA }\end{array}$} & \multirow[t]{2}{*}{$\begin{array}{l}\text { \% of reduction } \\
\text { with } 30 \% \mathrm{SA}\end{array}$} & \multicolumn{2}{|c|}{$\begin{array}{l}\text { Mean } \\
\text { standard deviation }\end{array}$} & \multirow[t]{2}{*}{$P$ value } \\
\hline & & & GA & SA & \\
\hline Comedones & 88.45 & 89.00 & $-0.88 \pm 0.15$ & $-0.90 \pm 0.13$ & 0.41 \\
\hline Papules & 88.65 & 90.36 & $-0.89 \pm 0.15$ & $-0.90 \pm 0.13$ & 0.53 \\
\hline Pustules & 89.62 & 84.87 & $-0.31 \pm 0.45$ & $-0.28 \pm 0.42$ & 0.673 \\
\hline Nodules/cysts & 83.33 & 100 & $-0.05 \pm 0.22$ & $-0.03 \pm 0.17$ & 0.47 \\
\hline
\end{tabular}

A similar study by Kessler et.al of glycolic and salicylic acid peels found similar efficacy of both peels on acne with salicylic acid to be showing fewer side effects [4].

\section{Discussion}

Alpha hydroxy acids and beta hydroxy acids are the most commonly used as peeling agents. Glycolic acid peels (AHA), thin the stratum corneum, promote epidermolysis and disperse basal layer melanin [5].

Salicylic acid is a beta hydroxyl acid agent which removes intracellular lipids that are covalently linked to the cornified epitheloid cells. The most efficacious concentration for acne scars in $30 \%$ in multiple session, 3-5 times, every 3-4 weeks $[6,7,8]$.

Acne vulgaris, the "Stigma of Adolescence" exceeds all other causes of suffering in adolescence age group. Many patients do not seek physician's advice. Only a few cosmetically conscious adolescents who are to be married shortly came for treatment, however mild the condition may be.

Acne therapy aims at reduction of sebum production, correcting the abnormal ductal keratinisation, reducing the colony of propionibacterium acnes and preventing the release of inflammatory mediators that are basically responsible for the pathogenesis of acne.

Age and sex distribution- In our study maximum number of patients of acne vulgaris (52\%) were between 21-25 years of age as more were the college students. with earlier observations as reported by Atzori L et al (1999) [9] where maximum patient were in age group 13-40 years commoner being the females, in our study also there was female preponderance being $64 \%$ female out of 200 patients.

$35 \%$ glycolic acid group: Patients in this group showed a good response in both inflammatory and noninflammatory lesions at the end of treatment. At the end of treatment comedones, papules and pustules showed $88.45 \%, \quad 88.65 \%$ and $89.62 \%$ improvement respectively. Various studies showing good results of glycolic acid on all the different types of acne lesions have been documented. Wang et al reported $90 \%$ improvement of overall acne lesions with glycolic acid Grover $\mathrm{C}$ et al reported good response in $78 \%$ patients[10]. Wang et al also observed $69 \%$ improvement in scarring and hyperpigmentation.

In this study we observed mild to moderate improvement in hyperpigmentation and moderate to good improvement in acne scarring with glycolic acid. The side effects such as erythema, burning and peeling of skin and dryness which have been seen in all patients in the present study have also been noted in the literature. These side effects were mild to moderate type and were well tolerated by patients. Not a single patient 
complained of severe side effects. No patient developed inflammatory hyperpigmentation or scarring or allergic reaction.

$30 \%$ Salicylic Acid Group: In this group reduction of both inflammatory and non-inflammatory lesions was better than glycolic acid. Comedones showed $89.6 \%$ improvement whereas papules had slightly better improvement $(90.36 \%)$ than in glycolic acid group. The basis of this is salicylic acid inhibit microcomedones formation, thus leading to decrease in follicular openings [11]. Salicylic acid showed better result than glycolic acid in treating pustules. They were decreased by $94.83 \%$. In a study conducted by Grimes, salicylic acid peel on acne showed an excellent to moderate response in $88 \%$ and mild clearance in $12 \%$ [12].

Side effects were noted in all the patients. Erythema, burning, dryness and peeling of skin were severe. The side effects were more in salicylic acid than glycolic acid. No patient developed inflammatory hyperpigmentation or scarring or allergic reaction. Application of mild steroids and emollients resulted in reduction of the side effects.

In this study salicylic acid was better than glycolic acid in treating comedones, papules and pustules. This is because of the unique lipophilic and is hydrophilic, which makes it a weaker comedolytic agent. Furthermore it doesn't have anti-inflammatory properties of salicylic acid [11]. Both the agents were effective for post-acne hyperpigmentation. The same observations have been reported by Vijay Kumar Garg et al [11].

Salicylic acid improves post acne hyperpigmentation by slightly different mechanisms than GA peels. Salicylic acid is anti-inflammatory and thus serves to decrease existing post acne hyperpigmentation and prevent further inflammation. In addition, some studies found salicylic acid to have a whitening effect on the skin. This effect was seen in present study also.

\section{Conclusion}

Both, Glycolic acid and Salicylic acid are effective in treatment of acne vulgaris, post acne scarring and hyperpigmentation.

Glycolic acid and salicylic acid peels additionally decrease superficial scarring and improve skin texture.
Both the acids were tolerated well in both immediate and post peel period. However erythema, burning, dryness and peeling were seen more with salicylic acid peel but these complications were temporary.

On the basis of lesion counts and overall visual scale improvement, glycolic acid and salicylic acid both are effective agents as monotherapy in the treatment of acne. However, cost of therapy and frequent visits might be a limiting factor to patient compliance and treatment adherence.

Funding: Nil, Conflict of interest: None initiated, Permission from IRB: Yes

\section{References}

1. Saraf V: Chemical Rejuvination of the face of non facial areas in Asian skin.1st edition 2003, Page no 5 ( https://books.google.co.in/books)

2. Conellessa C,Peris K, Onorati M T, Fangnoli MC, Chemiti, The use of chemical peeling in the treatment of different cutaneous hyperpigmentation, Dermatol surg. 1999 June 25 (6) 50-4.

3. Edward Kessler, BA, Katherine Flanagan, Christina Chia, Cynthia Rogers, and Dee Anna Glaser : Comparison of a- and b-Hydroxy Acid chemical peels in the treatment of mild to moderately severe facial acne vulgaris, dermatol surg. 2008;34:45-51.

4. Grover C, Reddu BS, The therapeutic value of glycolic acid peels in dermatology, IJDVL, 2003; $69 ; 2 ; 148-150$

5. Bernstein EF, Lee J, Brown DB. Glycolic acid treatment increases type 1 collagen mRNA and Hyaluronic acid content of human skin. Dermatol Surg2001;27:429-33.

6. Lazo ND, Meine JG, Downing DT. Lipids are covalently attached to right corneocyte protein envelopes existing predominantly as B-sheet: a solidstate nuclear magnetic resonance study. J Invest Dermatol 1995;105(2):296-300

7. Swinehart JM. Salicylic acid ointment peeling of the hands and forearms: effective nonsurgical removal of pigmented lesions and actinic damage. J DermatolSurg and Oncol1992;18(6):495-8. 
8. Imayama S, Ueda S, Isoda M. Histologic changes in the skin of hairless mice following peeling with salicylic acid. Archives of Dermatology 2000;136(11):1390-95

9. Atzori L(1), Brundu MA, Orru A, Biggio P. Glycolic acid peeling in the treatment of acne. J Eur Acad Dermatol Venereol. 1999 Mar;12(2):119-22.

10) Grover C, Reddu BS, The therapeutic value of glycolic acid peels in dermatology, IJDVL, 2003;69;2;148-150.
11. Garg VK, Sinha S, Sarkar R, Glycolic acid peels versus salicylic-mandelic acid peels in active acne vulgaris and post-acne scarring and hyperpigmentation: a comparative study. Dermatol Surg. 2009 Jan;35(1):59-65. doi: 10.1111/j.15244725.2008.34383.x. Epub 2008 Dec 8

12. Pearl E. Grimes, MD, The Safety and Efficacy of Salicylic AcidChemical Peels in Darker Racial- Ethnic Groups. Dermatol Surg.1999; 25:18-22.

\section{How to cite this article?}

Sharma P, Shah A, Dhillon AS. Study of glycolic acid and salicylic acid peels as a sole therapy in treatment of acne vulgaris. Int J Med Res Rev 2016;4(12):2205-2210.doi:10.17511 /ijmrr. 2016.i12.21. 